\title{
Biodentine Total Pulpotomy as an Effective Alternative to Conventional Endodontic Therapy for Young Permanent Teeth - A Study from Calicut, India
}

\author{
Amith Adyanthaya ${ }^{1}$, Prathyusha $\mathrm{P}^{2}$, Risana $\mathrm{K}^{3}$, Aparna Sivaraman ${ }^{4}$, \\ Nazreen Ayub K ${ }^{5}$, Reshma Aloysious ${ }^{6}$, Sangeetha C. R. ${ }^{7}$, Swetha S. Nair ${ }^{8}$ \\ 1, 2, 3, 4, 5, 6, 7, 8 Department of Paediatric and Preventive Dentistry, KMCT Dental College, Calicut, Kerala, India.
}

\section{ABSTRACT}

\section{BACKGROUND}

The vitality of dental pulp is essential for long-term survival of the tooth. Vital pulp therapy (VPT) intends to maintain healthy pulp tissue by eliminating bacteria from the dentin-pulp complex. There are many treatment options for vital pulp therapy in extensively decayed teeth. Pulp capping or pulpotomy procedures rely upon an accurate assessment of the pulp status, and careful management of the remaining pulp tissue. We wanted to evaluate as to whether biodentine total pulpotomy is an effective alternative to conventional endodontic therapy for young permanent teeth.

\section{METHODS}

Full coronal pulpotomy was performed in young permanent molars diagnosed with acute irreversible pulpitis with Biodentine as the pulpotomy medicament. Follow up evaluation was done clinically and radiographically at 3, 6 and 12 months.

\section{RESULTS}

During follow-up periods clinical signs/symptoms were absent including pulpal pain, swelling or presence of sinus and percussion pain. Continuous root development and healing of periapical radiolucency were noticed in the study which were indicative of maintenance of vitality of the pulp.

\section{CONCLUSIONS}

Within the limitations of this clinical study, it is concluded that total pulpotomy with Biodentine has a promising scope in regenerative approaches in the treatment of carious young permanent teeth.

\section{KEY WORDS}

Biodentine, Coronal Pulpotomy, Permanent Teeth, Total Pulpotomy
Corresponding Author:

Dr. Risana $K$

Department of Paediatric and

Preventive Dentistry,

KMCT Dental College,

Mukkam, Calicut,

Kerala, India.

E-mail: risanarahoof@gmail.com

DOI: $10.14260 / j e m d s / 2021 / 332$

How to Cite This Article:

Adyanathaya A, Prathyusha P, Risana K, et al. Biodentine total pulpotomy as an effective alternative to conventional endodontic therapy for young permanent teeth - a study from Calicut, India. J Evolution Med Dent Sci 2021;10(21):15931597, DOI: 10.14260/jemds/2021/332

Submission 15-01-2021,

Peer Review 21-03-2021,

Acceptance 27-03-2021,

Published 24-05-2021.

Copyright (c) 2021 Amith Adyanthaya et al. This is an open access article distributed under Creative Commons Attribution License [Attribution 4.0 International (CC BY 4.0)] 


\section{BACKGROUND}

Endodontic treatment is a fairly predictable treatment option in dentistry with reported success rates of up to $86-98 \%{ }^{1}$ The objective of endodontic treatment is thorough debridement and cleaning of the root canal system of any infected pulp tissue so the canal space can be shaped and prepared to be filled with an inert material thus preventing or minimizing any chances of reinfection. Endodontic procedures are always challenging for a practitioner despite the development of many advanced techniques and materials. ${ }^{2}$ The reasons are mainly the multiple variations in canal morphology and subjective errors that can rarely be eliminated.

There has not been a consensus in the literature upon a consistent definition of "success" criteria of endodontic treatment. An endodontically treated tooth should be evaluated clinically as well as radiographically for its root canal treatment to be deemed successful. The patient should be scheduled for follow-up visits to ascertain that the treatment is a success and the tooth in question is functional.

The endodontic and prosthodontic literature contains repeated references to the widely held clinical perception that endodontic treatment weakens teeth, resulting in increased brittleness. While Rosen ${ }^{3}$ described the dentin of endodontically treated teeth as "desiccated and inelastic," Johnson et al. ${ }^{4}$ additionally speculated that the elasticity of dentin decreased with time following the endodontic treatment. In conventional endodontic therapy, the removal of a substantial amount of dentin while performing the biomechanical preparation makes the tooth weaker and more prone to frequent fractures and subsequent loss of teeth. Alternatively, it has been suggested that, rather than endodontic treatment, loss of tooth structure associated with restorative procedures also act as a major factor in weakening teeth. While conventional root canal therapy asks for complete pulp tissue removal, less invasive methods have been described in the field of regenerative endodontics for the treatment of deep carious lesions.

Vital pulp therapy (VPT) is defined as a treatment which intends to preserve and maintain pulp tissue that has been compromised but not destroyed by caries, trauma, or restorative procedures in a healthy state. A full coronal pulpotomy is a vital pulp therapy indicating the removal of only the coronal pulp up to the level of canal orifices which is then followed by the placement of a suitable biocompatible material. This will protect the radicular pulp from further insult and will initiate healing and repair. ${ }^{5}$

Benefits of preservation of vital pulp includes the protective resistance to masticatory forces compared with a root-canal-filled tooth. Another major advantage of conservative treatment is the preservation of vital pulp including the defensive and proprioceptive functions. Maintaining the vitality of tooth in an immature permanent tooth will facilitate apexogenesis and root end closure. This will help in the attainment of adequate thickness of dentin and in turn help in improving the mechanical properties of the tooth.

Coronal pulpotomy for permanent teeth has progressively been declined in the literature because of the non-availability of ideal radicular pulp-capping materials. With the development of newer materials and evidence of improved success rates, it's time to appropriately reconsider coronal pulpotomy as a viable treatment option for young permanent teeth.

We wanted to evaluate as to whether biodentine total pulpotomy is an effective alternative to conventional endodontic therapy for young permanent teeth.

\section{METHODS}

This is a cross-sectional study conducted in the Department of Paediatric and Preventive dentistry, KMCT dental college, Mukkam, Calicut. The duration of the study was 24 months ranging from January 2018 - June 2020. Twenty-five patients reported to the department for management of carious permanent molar with signs and symptoms of irreversible pulpitis were considered for the study. After considering the inclusion criteria 10 patients were allotted to the study.

Pain associated with irreversible pulpal damage is defined as an unprovoked pain or pain which gets exacerbated by cold stimuli which lasts for a few seconds to several hours. After clinical examination preoperative pulpal and the periapical diagnosis was established. Patients were subjected to percussion and electric pulp testing for assessment of pulp vitality.

\section{Inclusion Criteria}

- Young permanent tooth with deep carious lesion and subsequent pulp exposure.

- $\quad$ Signs of irreversible pulpitis.

- Absence of tenderness to percussion.

- Positive response to vitality testing.

- Absence of any associated periodontal disease for the concerned tooth.

- Parent consent for treatment and continued follow up until 12 months.

\section{Exclusion Criteria}

- Tooth with signs of pulp necrosis.

- Presence of a sinus tract or swelling.

- Tooth subjected to trauma previously.

- Abscessed tooth.

- Radiographic signs of external or internal resorption.

- Calcified root canals.

- $\quad$ Patients with any systemic diseases or known allergic to the materials used in the study.

Each patient and his/her parents were explained in detail about the risks of the treatment. Briefing about the advantages, disadvantages and complications of the procedure was done. Written informed consent was obtained from the parents for the procedure and for follow up visits which included radiographs and pulp vitality testing.

All the procedures were carried out by a single operator. The tooth was subjected to local anaesthesia with $2 \%$ lidocaine (1:80000 epinephrine). After achieving proper 
anaesthesia isolation of the filed was done by the rubber dam (Optra Dam, Ivoclar Vivadent). Removal of the carious lesion was done using a high-speed air motor handpiece with adequate water coolant. After gaining access to the pulp chamber the status of pulpal bleeding was assessed. Presence of pulpal bleeding on entry to the pulp chamber was considered as the most important inclusion criterion for this case series. Pulp chamber was deroofed and the coronal pulpal tissue was removed using sharp excavator till the canal orifices. Pulpal bleeding was controlled by the placement of sterile moist cotton pellet for about 3 - 5 minutes. Biodentine (Septodont, France) was mixed by strictly following the manufacturer's instructions. The mixed material was placed on the floor of the pulp chamber at the root canal orifices in a thickness of approximately $2-3 \mathrm{~mm}$.

After setting of Biodentine the cavity was sealed using Zinc oxide eugenol cement. Clinical symptoms were evaluated at the end of 24 hours and if negative the Zinc oxide cement in the pulp chamber was removed partially and was replaced with the permanent restoration.

All the patients involved in the study were continuously evaluated clinically and radiographically at the end of 3 months, 6 months and 12 months. After 12 months, follow up visits were scheduled every six months.

The outcome of the treatment was considered successful in the -

1. Absence of any reported clinical signs and symptoms including pain

2. Absence of tenderness to percussion,

3. Absence of sinus tract or swelling

4. Absence of any pathologies evident on radiographs taken at follow up visits including root resorption, new furcal lesions or signs of any periapical rarefaction.

\section{Statistical Analysis}

The Fisher exact test was used to compare the outcome between cases with different baseline characteristics; statistical significance was set at $\mathrm{P}<0.05$.

\section{RESULTS}

There was no statistically significant difference in the Fisher exact test concerning gender, age and the type of restoration used. In this clinical study, only 1 procedure was performed without any comparison group. Out of the 10 cases, all except one were reported successful. For the same reason, regression analysis was not appropriate in the followed recall periods.

Ten patients in the clinical study included 4 boys and 6 girls. Age of the patients included in the study ranged from 6 to 13 years. The mean age being 9.7 years. All the patients included in the study had deep caries with signs of irreversible pulpitis. After ensuring positive vitality result coronal pulpotomy was performed for the patients.

Clinical and radiographic examination during the follow up periods revealed that 9 out of 10 patients were asymptomatic throughout the follow-up periods. There were no reported clinical signs / symptoms including pain, swelling, presence of sinus and tenderness to percussion.

Patients with periapical radiolucency showed healing of the lesion over the follow-up periods. (Figure 1) No cases were reported with new periapical lesions on the follow-up radiographs. Patients with open apex showed continued root development with the closure of the apex which shows that the vitality of the tooth is maintained. (Figure 2)
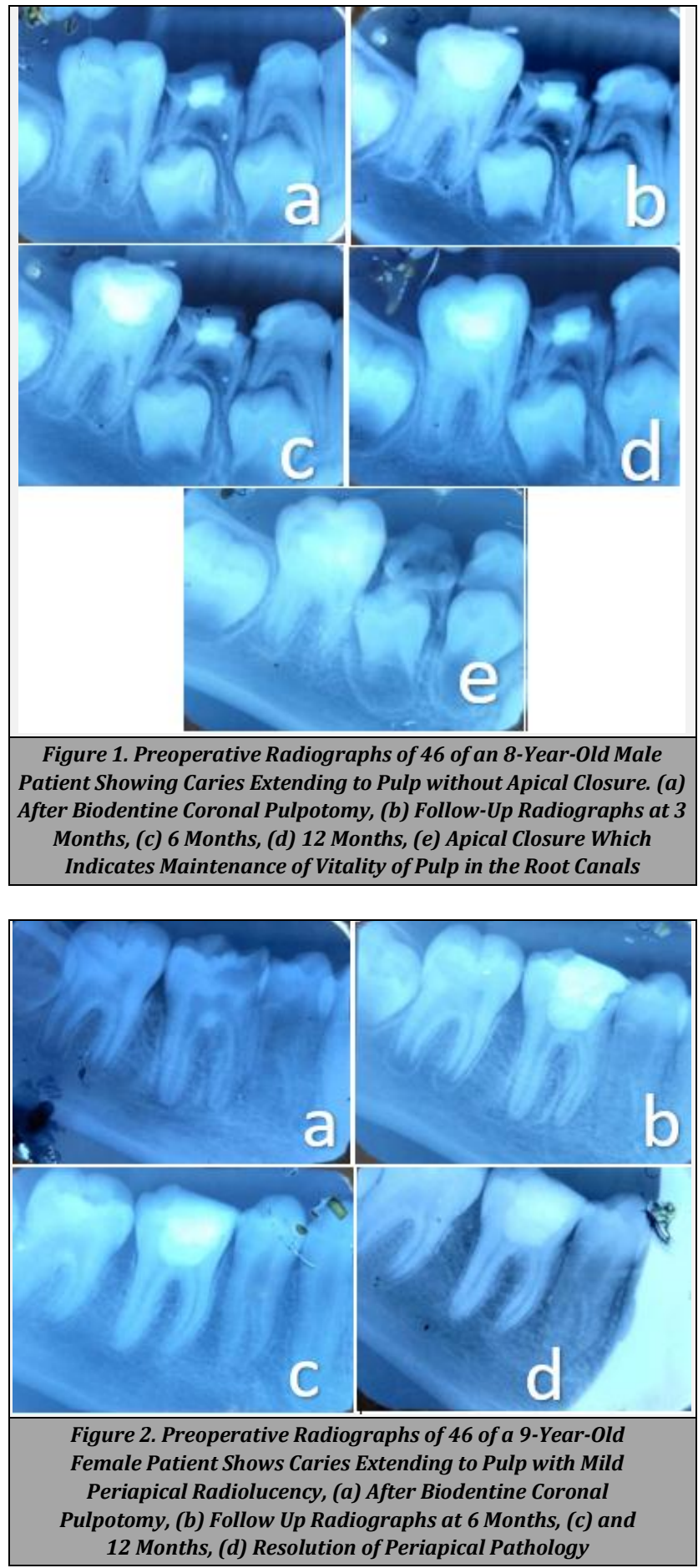

Only one patient complained of acute pain 1.5 months after the procedure and subsequently required root canal treatment. This case was considered as a clinical failure.

\section{DISCUSSION}

In endodontics, the treatment of teeth with carious pulp exposure is a controversial issue since long. Different point of 
views exists for the treatment of a tooth with deep carious lesion. This includes the conservative approach as in vital pulp therapy and the second approach includes more invasive approaches including procedures like pulpectomy and root canal therapy. Each treatment modality has its advantages and disadvantages in views of the patient and the practitioner.

From the point of view of a patient any procedure that relieves the clinical signs and symptoms and which prolongs the lifespan of a tooth is satisfactory and considered as successful. But in the view of a clinician and researcher besides resolution of the clinical signs and symptoms preservation of the functions of the pulp is considered as an important criterion for considering the treatment a real success.

The major criteria to decide the treatment of a carious tooth is to evaluate whether the pulp is damaged reversibly or irreversibly. 6 Pulp inflammation is a progressive disease which is initially confined to a small area. If the pulpal lesion is not arrested or treated in this stage it leads to the progression of inflammation to the whole pulp tissue and eventually to the root canals and ultimately to the periradicular tissues. ${ }^{7}$

The treatment of a diseased pulp in the initial stages is to remove the most severely inflamed and infected coronal pulp tissue followed by placement of a proper biocompatible pulp dressing material. A bioactive pulp dressing with healing ability can potentially conserve the vitality of the remaining healthy pulp tissues by promoting healing. On the contrary, if the pulpal tissue is inflamed beyond possible repair, treatment with partial removal of pulp will eventually fail. The patient will either present with the reappearance of the symptoms or they even development new periapical lesion.

For decades' calcium hydroxide has been considered as the material of choice in vital pulp therapy. A success rate of 86.7 $\%$ is reported for Calcium hydroxide in pulpotomy of immature permanent teeth by Meligy et al. ${ }^{8}$ Dissolution and degradation in oral and tissue fluids and failure in flexure were some reported disadvantages of calcium hydroxide. Moreover, the hard tissue barrier formed by calcium hydroxide is usually of poor quality. Mineral trioxide aggregate (MTA) was an alternate material recommended for use in pulpotomy due to its better sealing ability, marginal adaptation and biocompatibility. Initiation of hard tissue formation by MTA occurs by controlling infection via high alkalinity thus denaturing bacterial proteins and MTA also releases calcium and hydroxyl ions for the formation of the calcific barrier. But the major drawback of MTA is the prolonged setting time which is not desirable in clinical practice.

Biodentine (Septodont, France) is a calcium silicate cement which has comparable biocompatibility and capability of inducing the formation of a calcific barrier. Biodentine can increase the release of calcium and Transforming growth factor (TGF) - $ß 1$ from pulp cells which can eventually induce angiogenesis, cell differentiation and mineralization. ${ }^{9}$ It can induce odontoblast differentiation, odontoblast proliferation, migration of the odontoblasts and human pulp stem cells adhesion. These features make Biodentine a superior pulp capping material compared to calcium hydroxide and MTA.

Consideration of the interplay of inflammation in pulpal tissues helps in appropriate case selection and subsequent treatment planning in patients with signs and symptoms of irreversible pulpal damage.

Caries progression into deeper tissues of the pulp can cause degenerative and inflammatory processes resulting in necrosis of the pulp and apical periodontitis. In a carious pulp exposure, there is a coexistence of damaged and healthy pulp histologically in various parts within the same pulp. ${ }^{10}$ Proper evaluation of the extent of tissue injury of the pulp plays a major role in the successful prediction of pulpotomy treatment. The intensity of bacterial infection influences the inflammatory and immune response also. Clinically, it is not easy to accurately determine the extent of disease progression in the pulp tissue and hence, removal of the diseased tissue may not be complete and can contribute to the failure of such cases. ${ }^{11}$

Adoption of vital pulp therapy is depended upon a thorough understanding of the pulp biology and the regenerative and healing potential of the remaining pulp tissue. Actual histologic features and potential capability of the pulp to heal can be in a way predicted by the clinical signs and symptoms like the degree of pain. ${ }^{12}$ Matsuo et al. suggested that observing the degree of pulpal bleeding was more reliable than preoperative clinical signs and symptoms in providing a better idea of the histologic condition of the pulp. If the pulpal bleeding presents to be controllable it is indicative of a mild inflammation or chronic partial pulpitis without areas of necrosis. Meanwhile, if the pulp presents with profuse bleeding which appears difficult to control the pulp tissue it is assumed to be severely inflamed. ${ }^{13}$

There are various studies which have used the "time to stop bleeding" as a cut-off parameter to discriminate reversible pulpitis from irreversible pulpitis. The pulp is considered severely inflamed if the pulpal bleeding could not be stopped within 5 to 10 minutes. In such cases, partial removal of pulp is not recommended. But there have not been any thorough investigations carried out to evaluate the association between pulpal bleeding time and the degree of pulpal inflammation. Literature suggests various methods for controlling pulpal haemorrhage. This includes the use of sodium hypochlorite solution, ${ }^{14}$ physiologic salines, ${ }^{15}$ hydrogen peroxide or gentle pressure with a sterile moist cotton pellet. ${ }^{16}$ But there is no published data available to suggest the superiority of any of these techniques in clinical outcome compared to others. However, if the bleeding cannot be stopped within 5-10 minutes it suggests that the clinician has failed in removing the entire inflamed pulp or the inflammation of the pulp has progressed to the deeper radicular pulp. ${ }^{17}$ In such cases the treatment plan should be modified to pulpectomy rather than pulpotomy.

Vital pulp therapy in permanent teeth with cariously exposed pulp is a matter of controversies in the literature. Some researchers advocate vital pulp treatment only in young patients as pulp of young patients is believed to have a higher healing capacity. Pulp tissue of the older individuals will be more fibrous in nature. It exhibits reduced ability to overcome any insult as they possess less healing potential owing to the reduced cellularity and limited blood supply. These factors will affect the treatment outcomes in treatment of older patients. But this is also a matter of controversy.

Even though Aguilar and Linsuwanont ${ }^{18}$ observed that younger patients have a higher success in vital pulp therapy there are reports of successful vital pulp therapy in patients as young as 6 years to 70 years. This throws light to the fact that the healing capacity of pulp is present even in older patients if there is proper removal of the cause of the disease. This is explained to be because of subjective variation in the innate 
capacity of pulp tissue for repair in the absence of microbial contamination. Aguilar and Linsuwanont ${ }^{19}$ have reported 99.4 $\%$ and $99.3 \%$ success rate for partial pulpotomy in vital permanent teeth with closed apices.

As reported by Massler most of the failures in vital pulp therapy and endodontic treatment were attributed to microleakage at the coronal tooth restoration interface. Establishment of proper coronal seal serves as an important determinant in the success of vital pulp therapy. It reduces microleakage and bacterial invasion which will hamper the healing of dental pulp. Placement of an immediate full coronal restoration will greatly improve the chances of long-term success of vital pulp therapy.

Definite success factors for treatment outcome of a pulpotomy is not published in any review yet. Most of the published data is limited to clinical studies with a smaller number of participants. There is a proven association between delay in placement of restoration and unfavourable outcomes. Hence, in this study permanent restorations were performed within 24 hours after the coronal pulpotomy.

Another important factor which can cause the failure of pulpotomy treatment is the bacterial contamination of the pulp while accessing the chamber for the removal of the coronal pulp tissue. The high success rate in this case series is attributed to the careful attention given to the case selection, maintenance of proper isolation and to the methods used to prevent pulpal bleeding and cavity closure.

\section{CONCLUSIONS}

Within the limitations, the present study showed that Biodentine can be used as an effective medicament for total pulpotomy of young permanent teeth with reported signs and symptoms of irreversible pulpal damage. Biodentine effectively relieved the associated pulpal symptoms and was successful in maintaining the pulp vitality. Henceforth coronal pulpotomy shall be considered as an option for treatment of permanent teeth. Although the results are in favour of coronal pulpotomy as a treatment of the permanent tooth, the literature calls for clinical trials with larger sample size and a longer follow up period to justify the use of Biodentine for treatment of young permanent teeth.

Data sharing statement provided by the authors is available with the full text of this article at jemds.com.

Financial or other competing interests: None.

Disclosure forms provided by the authors are available with the full text of this article at jemds.com.

\section{REFERENCES}

[1] Song M, Kim HC, Lee W, et al.. Analysis of the cause of failure in nonsurgical endodontic treatment by microscopic inspection during endodontic microsurgery. J Endod 2011;37(11):1516-9.
[2] Soni HK. Biodentine pulpotomy in mature permanent molar: a case report. J Clin Diagn Res 2016;10(7):ZD0911.

[3] Rosen H. Operative procedures on mutilated endodontically treated teeth. J Prosthet Dent 1961;11(5):972-86.

[4] Johnson JK, Schwartz NL, Blackwell RT. Evaluation and restoration of endodontically treated posterior teeth. J Am dent Assoc 1976;93(3):597-603.

[5] Farges JC, Alliot-Licht B, Renard E, et al.. Dental pulp defence and repair mechanisms in dental caries. Mediators Inflamm 2015;2015:230251.

[6] Barngkgei IH, Halboub ES, Alboni RS. Pulpotomy of symptomatic permanent teeth with carious exposure using mineral trioxide aggregate. Iran Endod J 2013;8(2):65-8.

[7] Taha NA, Ahmad MB, Ghanim A. Assessment of mineral trioxide aggregate pulpotomy in mature permanent teeth with carious exposures. Int Endod J 2017;50(2):117-25.

[8] El-Meligy OAS, Avery DR. Comparison of mineral trioxide aggregate and calcium hydroxide as pulpotomy agents in young permanent teeth (apexogenesis). Pediatr Dent 2006;28(5):399-404.

[9] Torabinejad M, Parirokh M, Dummer PMH. Mineral trioxide aggregate and other bioactive endodontic cements: an updated overview-part II: other clinical applications and complications. Int Endod J 2018;51(3):284-317.

[10] Simon S, Perard M, Zanini M, et al.. Should pulp chamber pulpotomy be seen as a permanent treatment? Some preliminary thoughts. Int Endod J 2013;46(1):79-87.

[11] Eghbal MJ, Asgary S, Baglue RA, et al.. MTA pulpotomy of human permanent molars with irreversible pulpitis. Aust Endod J 2009;35(1):4-8.

[12] Bjørndal L. The caries process and its effect on the pulp: the science is changing and so is our understanding. J Endod 2008;30(3):192-6.

[13] Matsuo T, Nakanishi T, Shimizu H, et al.. A clinical study of direct pulp capping applied to carious-exposed pulps. J Endod 1996;22(10):551-6.

[14] Bogen G, Kim JS, Bakland LK. Direct pulp capping with mineral trioxide aggregate: an observational study. J Am Dent Assoc 2008;139(3):305-15.

[15] Mejàre I, Cvek M. Partial pulpotomy in young permanent teeth with deep carious lesions. Endod Dent Traumatol 1993;9(6):238-42.

[16] Caliskan MK. Success of pulpotomy in the management of hyperplastic pulpitis. Int Endod J 1993;26(2):142-8.

[17] Mass E, Zilberman U. Clinical and radiographic evaluation of partial pulpotomy in carious exposure of permanent molars. Pediatr Dent 1993;15(4):257-9.

[18] Aguilar P, Linsuwanont P. Vital pulp therapy in vital permanent teeth with cariously exposed pulp: a systematic review. J Endod 2011;37(5):581-7.

[19] Linsuwanont P, Wimonsutthikul K, Pothimoke U, et al.. Treatment outcomes of mineral trioxide aggregate pulpotomy in vital permanent teeth with carious pulp exposure: the retrospective study. J Endod 2017;43(2):225-30. 